\title{
Estrogen enhances vasoconstrictive remodeling after injury in male rabbits
}

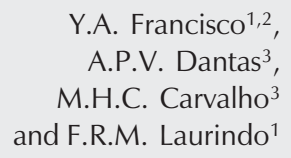

Y.A. Francisco ${ }^{1,2}$,

A.P.V. Dantas ${ }^{3}$,

M.H.C. Carvalho ${ }^{3}$ and F.R.M. Laurindo ${ }^{1}$

\author{
${ }^{1}$ Laboratórios de Biologia Vascular e Fisiologia Aplicada, Instituto do Coração, \\ ${ }^{2}$ Departamento de Medicina de Emergência, Faculdade de Medicina, and \\ ${ }^{3}$ Departamento de Farmacologia, Instituto de Ciências Biomédicas, \\ Universidade de São Paulo, São Paulo, SP, Brasil
}

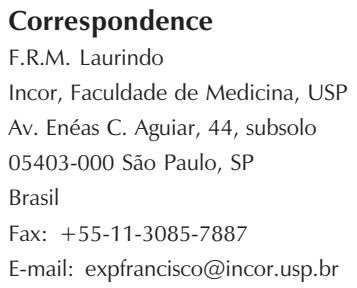

Abstract

The complete spectrum of estrogen vascular effects remains unclear. In particular, estrogen effects in the vascular response to profound injury in males have not been explored in detail. Therefore, we submitted 44 male New Zealand rabbits weighing $3.4 \pm 0.6 \mathrm{~kg}$ to overdistention balloon injury of the right iliac artery. Rabbits were given $17 ß$-estradiol $(5.45 \mu \mathrm{mol} /$ day, $s c)$ or vehicle for 7 days before and 14 days after injury, when the arteries were examined by postmortem histomorphometry. Arteriographic caliber was assessed in vivo at baseline and before sacrifice. On day 14 after injury, in vivo arteriographic caliber (baseline $=2.44 \pm 0.43 \mathrm{~mm}$ ) was decreased by $23.1 \pm 0.1 \%$ in controls and by $44.5 \pm 0.1 \%$ in estrogen-treated rabbits $(\mathrm{P}<0.001)$. Neither the neointimal area nor the neointima/media area ratio changed after estrogen treatment. Collagen fraction was increased in the media and neointima of estrogen-treated rabbits $v s$ control $(1.38 \pm 1.30$ vs $0.35 \pm 0.67$, respectively, $\mathrm{P}=0.01)$. Taken together, these findings suggest that estrogen increased negative vascular remodeling. Transcription of endothelial and inducible nitric oxide synthases (eNOS and iNOS) was analyzed by RT-PCR. eNOS mRNA expression was marginally increased after estrogen $(\mathrm{P}=0.07)$ and injury. iNOS mRNA was increased 2- to 3-fold on day 14 after injury. With estrogen treatment, iNOS mRNA increased in uninjured arteries and exhibited a further 5.5-fold increase after injury. We concluded that estrogen increased lumen loss after balloon injury in male rabbits, likely by increased negative remodeling, which may be related to increased iNOS transcriptional rates.
Key words

- Estrogen

- Restenosis post-angioplasty

- Nitric oxide

- Endothelial function

- Vascular remodeling
Endothelial dysfunction underlies the pathogenesis of several vascular diseases, in particular the vascular response to angioplasty, which often culminates in restenosis (1). A common feature of these vascular processes is loss of nitric oxide (NO) bioavailability and generation of oxidative/ nitrative intermediates (2). Estrogen hormones exhibit several actions favoring normalization of endothelial function, reduction of atheroma formation and particularly inhibition of neointimal growth after injury in female rats (3) and pigs (4). Given the wealth of experimental data indicating the beneficial effects of estrogen, the possible therapeutic uses of estrogen continue to be a relevant topic despite the contradictory results of clinical trials. In particular, the clinical use of estrogen in males is potentially important (5), especially because of local 
delivery (6), but the effects of estrogen on males after vascular injury are poorly known. Our objective was to examine the effects of estrogen on neointimal growth and vascular caliber after iliac artery injury induced with a balloon catheter in male rabbits.

The study was approved by the institutional Scientific Ethics Committee and conformed to the Guidelines for Care and Use of Laboratory Animals of the National Institutes of Health (NIH Publication \#85-23, reviewed in 1996).

Arterial injury was induced as described previously (1) by overdistention (8 atm) of a coronary-type balloon angioplasty catheter (3.0 $\mathrm{mm}$ in diameter) in the right iliac artery of 44 intact male New Zealand white rabbits weighing $3.4 \pm 0.6 \mathrm{~kg}$. Arteriography was performed through a carotid access by injection of $3 \mathrm{ml}$ iopamidol (Schering, São Paulo, SP, Brazil) into the lower abdominal aorta, allowing simultaneous visualization of the injured and the contralateral non-injured iliac arteries. Digital images were acquired with a DVI-Super 80 Philips ${ }^{\circledR}$, Koninklijke Philips Electronics N.V., The Netherlands) on standard film. Correction factors were calculated using a calibrated intra-aortic guidewire. Baseline arteriography was performed 7 days before arterial injury, and each animal was assigned to a group to receive either estrogen or vehicle. Fourteen days post-injury (PI), a second arteriogram was performed and the rabbits immediately killed with pentobarbital.

Iliac arteries were perfusion-fixed ex-vivo in situ, at $100 \mathrm{mmHg}$ with $10 \%$ formaldehyde in PBS, $\mathrm{pH} \mathrm{7.4,} \mathrm{for} 60 \mathrm{~min}$, followed by immersion fixation for another $48 \mathrm{~h}$. The segments between the hypogastric and femoral profundis branches were then cut into 4- to 5$\mathrm{mm}$ long rings, which were embedded in paraffin and stained by the Verhoeff-van-Gieson method and with hematoxylin-eosin. Collagen content was assessed by the Picrosyrius method; the amount of polarized light was scored semiquantitatively as grade $0-3$ by a blinded observer. Histomorphometric analy- sis was performed with Leica Quantimet (Cambridge, England) software. Arteriographic images were divided into 6 segments matching as much as possible the ones for histomorphometry. The average caliber of each segment was measured with a caliper.

Rabbits received $s c$ either 17ß-estradiol (Sigma, St. Louis, MO, USA; $5.45 \mu \mathrm{mol}$ daily) or vehicle ( $0.5 \mathrm{ml}$ almond oil) for 21 days (7 days before angioplasty and 14 days PI). The choice of the dose was based on prior studies using a rabbit atherosclerosis model (7).

RT-PCR analysis of nitric oxide synthase (NOS) mRNA was performed as described by Ferreiro et al. (8). Data are reported as means \pm SD for rabbits that survived all stages of the study. Comparison between means or proportions was performed by the $t$-test or $\chi^{2}$ test, respectively (9), with the level of significance set at $\mathrm{P}<0.05$.

Figure 1A depicts the changes in arteriographic calibers from baseline to 14 days PI measured in vivo just before sacrifice. In vehicle-treated rabbits, lumen caliber was decreased by $24.2 \%$, a value within the range observed previously in analogous experiments from our laboratory (1). In contrast, lumen caliber was decreased by $38.5 \%$ in estrogen-treated rabbits $(\mathrm{P}<0.001 v s$ vehicle). This effect was also significant when data were expressed as percent lumen loss vs uninjured contralateral artery (data not shown). Panel B shows the values of absolute lumen diameters. The lumen diameters of the contralateral iliac arteries were not significantly different.

Histomorphometric analysis showed similar neointimal size for vehicle- and estrogen-treated rabbits, whether analyzed as absolute neointimal, medial or total wall areas or as intima/media area ratio. The latter was $0.63 \pm 0.8$ after vehicle and $0.60 \pm 0.46$ after estrogen $(\mathrm{P}=\mathrm{NS})$.

There was an increase in collagen accumulation in the media and neointima of estrogen-treated rabbits $v s$ control $(\mathrm{N}=5$ each 


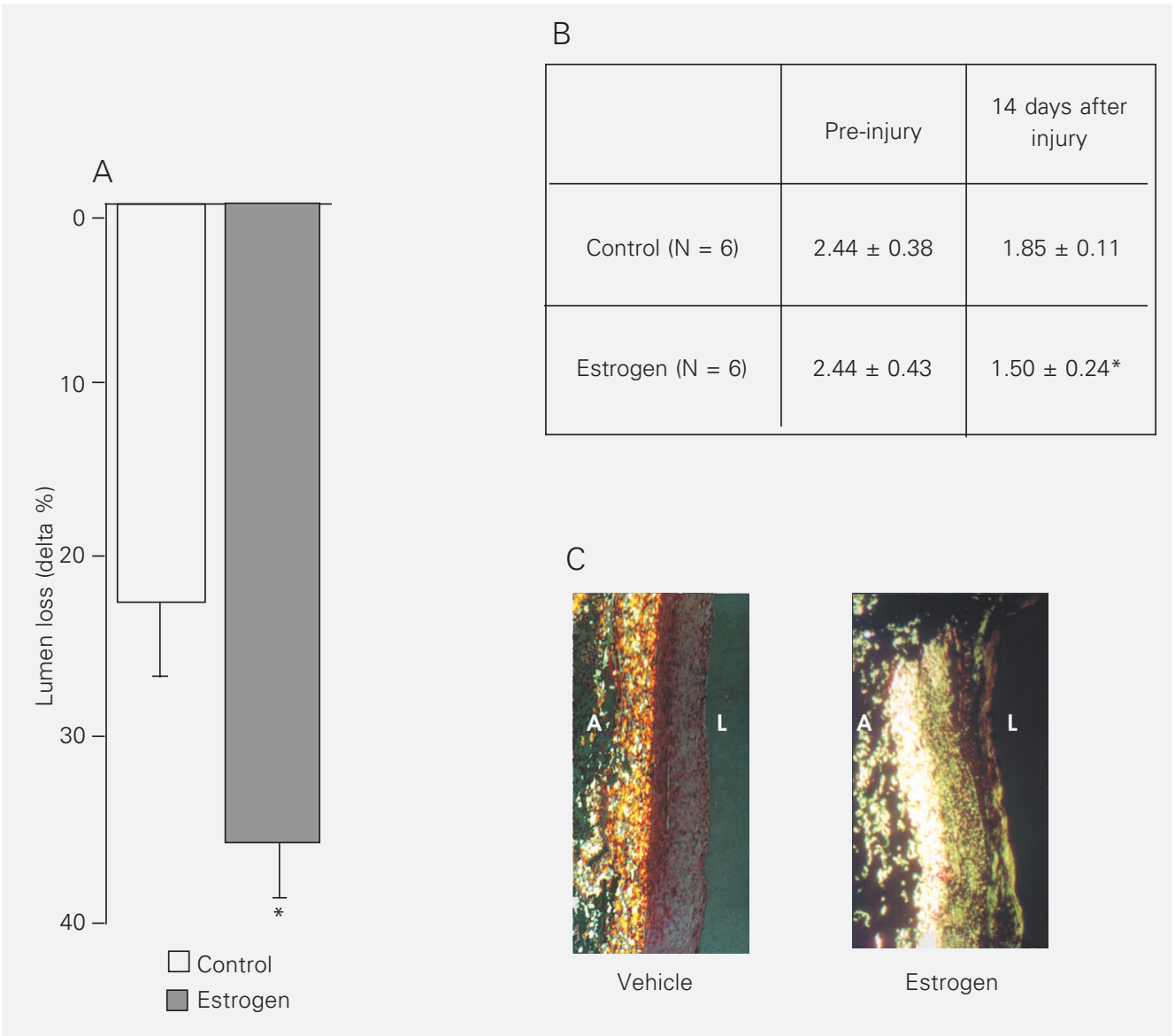

group; respective semi-quantitative scores were $1.38 \pm 1.30$ vs $0.35 \pm 0.67, \mathrm{P}=0.01$ ) on day 14 PI, with a greenish-yellow pattern of polarized light suggesting newly synthesized collagen. In the adventitia, the strong orange/pink polarized light emission was not clearly different between groups.

Expression of endothelial (eNOS) and inducile NOS (iNOS) mRNA ( $\mathrm{N}=9$ rabbits) in iliac arteries 14 days PI or in uninjured controls is shown in Figure 2. eNOS mRNA expression was marginally increased after estrogen $(\mathrm{P}=0.07)$ and injury. iNOS mRNA expression was significantly increased by estrogen in uninjured arteries vs control. It is known that injury up-regulates iNOS expression (10) and a further up-regulation was observed with estrogen.

Exogenous administration of 17ß-estradiol to male rabbits submitted to iliac artery
Figure 1. Panel $A$, Bar graph depicting \% change in in vivo iliac artery lumen caliber in vehicleor estrogen-treated rabbits, $\mathrm{N}=$ 6 for each group; ${ }^{*} P<0.001$ vs control (t-test). Panel B, Absolute values of arteriographic caliber at baseline and 14 days after injury in vehicle- or estrogentreated rabbits; ${ }^{*} P=0.009$ vs pre-injury (Student paired $t$-test). Panel C, Representative Picrosyrius staining of the iliac artery 14 days after injury in vehicle- or estrogen-treated rabbits viewed under the polarized light microscope. $\mathrm{A}=$ adventitia; $\mathrm{L}=\mathrm{lu}-$ men.

Figure 2. Densitometric analysis of RT-PCR-amplified mRNA levels for eNOS (four left columns) and iNOS (four right columns) in uninjured control or 14 days after balloon injury of iliac arteries from rabbits treated with vehicle (open columns) or estrogen (filled columns). eNOS = endothelial nitric oxide synthase; iNOS = inducible nitric oxide synthase. 
caliber loss after balloon injury, as reported in experimental histopathological studies on rabbits (1) and pigs (6), as well as in ultrasound studies on man (11). Although our model offers valuable insights into some aspects of restenosis and vascular pathophysiology, extrapolations to clinical postangioplasty restenosis should be considered with caution. First, ours is essentially a model of vascular scar formation, which is only one component of the more complex restenosis process. Second, the inflammatory process of human atheroma, not fully represented in our model, could modify vascular repair (12). The correlation between collagen vessel wall content and constrictive remodeling has been described previously (13) and is in accordance with our findings.

Estrogen therapy is known to decrease neointimal growth in female animal models through mechanisms involving inhibition of smooth muscle cell proliferation (4), reduced $\beta_{3}$ integrin-dependent migration of adventitial fibroblasts or increase in endothelial proliferation. The distinct result in our study may have been due to type of injury, arterial bed and especially animal gender, the latter two factors affecting both the type and density of estrogen receptors (14). The attempt to measure estrogen receptor alpha was not successful, possibly due to its reduced density in male vascular tissue. It is conceivable that a potential improvement of endothelial function due to estrogens may have been buffered by a number of complex counteracting actions (15), a possibility in line with the disparity between the known experimental estrogen effects and their less evident clinical benefit (16).

Although prior studies showed that iNOS transfection reduces neointimal growth (15), the simultaneous increases in iNOS message, lumen loss and collagen accumulation observed in the present study raise the hypothesis that iNOS activity may be involved in constrictive vascular remodeling. In agreement with our findings, in the unilateral carotidtying model, iNOS knock-out mice showed reduced constrictive remodeling without a change in neointima, which was decreased only in the eNOS knock-out mice (17). On the other hand, collagen accumulation is increased in atherosclerotic iNOS-/-mice (18). We speculate that iNOS may be protective up to the point when enzyme activity yields biologically active NO, but not in situations of excessive activity in which oxidizing or nitrating species are released. Thus, the most likely explanation for our findings is that increased NOS activity does not mean an increase in bioactive $\mathrm{NO}$ either because $\mathrm{NO}$ is being inactivated via increased superoxide production or because the enzyme itself is generating superoxide instead of NO (NOS uncoupling) (2). This proposal agrees with our recent findings that superoxide dismutase replenishment after injury decreases nitrotyrosine levels and rescues bioavailable nitric oxide from inducible nitric oxide synthase (19). Another report showed that micromolar, as opposed to nanomolar, estrogen concentrations used here led to vascular iNOS stimulation and deleterious effects (10). Although accurate estrogen levels could not be documented in our study due to profound interference with the immunoassay, our concentrations were safely below low nanomolar levels (consistent with the observed absence of detection by HPLC at the $\sim 1 \mu \mathrm{M}$ detection limit; data not shown). Even so, it is possible that male rabbits have increased susceptibility to iNOS induction, with resulting pro-inflammatory effects (20), given differences in estrogen receptors or even receptorindependent effects plus the setting of profound arterial injury (which by itself strongly induces iNOS protein) (19). Estrogens may enhance vascular caliber loss after injury in males. Such results may help understand some potentially deleterious effects of estrogen.

\section{Acknowledgments}

We are indebted to Leonora Loppnow for technical support and to Breno Almeida, $\mathrm{MD}$, for performing the angiograms. 


\section{References}

1. Laurindo FR, Souza HP, Pedro MA \& Janiszewski M (2002). Redox aspects of vascular response to injury. Methods in Enzymology, 352: 432-454.

2. Vasquez-Vivar J, Hogg N, Martasek P, Karoui H, Pritchard Jr KA \& Kalyanaraman B (1999). Tetrahydrobiopterin-dependent inhibition of superoxide generation from neuronal nitric oxide synthase. Journal of Biological Chemistry, 274: 26736-26742.

3. Tolbert T, Thompson JA, Bouchard P \& Oparil S (2001). Estrogen induced vasoprotection is independent of inducible nitric oxide synthase expression; evidence from the mouse carotid artery ligation model. Circulation, 104: 2740-2745.

4. Chandrasekar B \& Tanguay JF (2000). Local delivery of 17 betaestradiol decreases neointimal hyperplasia after coronary angioplasty in porcine model. Journal of the American College of Cardiology, 36: 1972-1978.

5. VanPottelberg I, Braeckman L, DeBascquer D, DeBacker G \& Kaufman JM (2003). Differential contribution of testosterone and estradiol in the determination of cholesterol and lipoprotein profile in healthy middle-aged men. Atherosclerosis, 166: 95-102.

6. New G, Moses JW, Roubin GS, Leon MB, Colombo A, lyer SS, Tio FO, Mehran R \& Kipshidze N (2002). Estrogen-eluting, phosphorylcholine-coated stent implantation is associated with reduced neointimal formation but no delay in vascular repair in a porcine coronary model. Catheterization and Cardiovascular Diagnosis, 57: 266-271.

7. Gorodeski G, Yang T, Levy MN, Goldfarb J \& Utian WH (1995). Effects of estrogen in vivo on coronary vascular resistance in perfused rabbit hearts. American Journal of Physiology, 269: H1333H1338.

8. Ferreiro $C R$, Chagas $A C$, Carvalho $M H$, Dantas $A P$, Jatene MB, Bento De Souza LC \& Lemos Da Luz P (2001). Influence of hypoxia on nitric oxide synthase activity and gene expression in children with congenital heart disease: a novel pathophysiological adaptive mechanism. Circulation, 103: 2272-2276.

9. Glantz SA (1992). Primer of Biostatistics. 3rd edn. McGraw Hill, Columbus, OH, USA.

10. Cho M, Ziats NP, Pal D, Utian WH \& Gorodeski GI (1999). Estrogen modulates paracellular permeability of human endothelial cells by
eNOS and iNOS related mechanisms. American Journal of Physiology, 276: C337-C349.

11. Heckenkamp J, Gawenda M \& Brunkwall J (2002). Vascular restenosis: Basic science and clinical implications. Journal of Cardiovascular Surgery, 43: 349-357.

12. Farsi A, Domeneghetti MP, Brunelli T et al. (2001). Activation of the immune system and coronary artery disease: the role of anti-endothelial cell antibodies. Atherosclerosis, 154: 429-436.

13. Rajagopal V \& Rockson SG (2003). Coronary restenosis: a review of mechanisms and management. American Journal of Medicine, 115: 547-553.

14. Mendelsohn ME (2000). Nongenomic, estrogen-mediated activation of endothelial nitric oxide synthase. Circulation Research, 87: 956-960.

15. Tostes RC, Nigro D, Fortes ZB \& Carvalho MHC (2003). Effects of estrogen on the vascular system. Brazilian Journal of Medical and Biological Research, 36: 1143-1158.

16. Herrington DM \& Howard TD (2003). From presumed benefit to potential harm - Hormone therapy and heart disease. New England Journal of Medicine, 349: 519-521.

17. Yogo K, Shimokawa H, Funakkoshi H, Kandabashi T, Miyata K, Okamoto S, Egashira K, Huang P, Akaike T \& Takeshita A (2000). Different vasculoprotective roles of $\mathrm{NO}$ synthase isoforms in vascular lesion formation in mice. Arteriosclerosis, Thrombosis, and Vascular Biology, 20: E96-E100.

18. Niu $X L$, Yang $X$, Hoshai $K$, Tanaka $K$, Sawamura $S$, Koga $Y$ \& Nakazawa H (2001). Inducible NOS deficiency does not affect the susceptibility of mice to atherosclerosis but increases collagen content in lesions. Circulation, 103: 1115-1120.

19. Leite PF, Danilovic A, Moriel P, Dantas K, Marklund S, Dantas AP \& Laurindo FR (2003). Sustained decrease in superoxide dismutase activity underlies constrictive remodeling after balloon injury in rabbits. Arteriosclerosis, Thrombosis, and Vascular Biology, 23: $2197-$ 2202.

20. Harris MT, Feldberg RS, Lau KM, Lazarus NH \& Cochrane DE (2000). Expression of proinflammatory genes during estrogen-induced inflammation of the rat prostate. Prostate, 44: 19-25. 